\title{
Singularities of Robot Manipulators
}

\author{
Peter Donelan \\ School of Mathematics, Statistics and Computer Science, \\ Victoria University of Wellington, \\ PO Box 600, Wellington, New Zealand. \\ peter.donelan@vuw.ac.nz
}

\begin{abstract}
Engineers have for some time known that singularities play a significant role in the design and control of robot manipulators. Singularities of the kinematic mapping, which determines the position of the end-effector in terms of the manipulator's joint variables, may impede control algorithms, lead to large joint velocities, forces and torques and reduce instantaneous mobility. However they can also enable fine control, and the singularities exhibited by trajectories of the points in the end-effector can be used to mechanical advantage.

A number of attempts have been made to understand kinematic singularities and, more specifically, singularities of robot manipulators, using aspects of the singularity theory of smooth maps. In this survey, we describe the mathematical framework for manipulator kinematics and some of the key results concerning singularities. A transversality theorem of Gibson and Hobbs asserts that, generically, kinematic mappings give rise to trajectories that display only singularity types up to a given codimension. However this result does not take into account the specific geometry of manipulator motions or, a fortiori, to a given class of manipulator. An alternative approach, using screw systems, provides more detailed information but also shows that practical manipulators may exhibit high codimension singularities in a stable way. This exemplifies the difficulties of tailoring singularity theory's emphasis on the generic with the specialized designs that play a key role in engineering.
\end{abstract}

Keywords: singularity, transversality, robot manipulator, screw system 


\section{Robot Manipulators}

The International Federation for the Promotion of Mechanism and Machine Science [47] has defined a mechanism to be "a system of bodies designed to convert motions of, and forces on, one or several bodies into constrained motions of, and forces on, other bodies"; a robot is a "mechanical system under automatic control that performs operations such as handling and automation", while a manipulator is a "device for gripping and the controlled movement of objects". More comprehensively, the International Organisation for Standardisation (ISO) [46] defines a manipulator to be "a machine, the mechanism of which usually consists of a series of segments, jointed or sliding relative to one another, for the purpose of grasping and/or moving objects (pieces or tools) usually in several degrees of freedom. It may be controlled by an operator, a programmable electronic controller, or any logic system (for example cam device, wired, etc.)". Certainly these definitions are necessarily imprecise, but we can construct succinct mathematical descriptions that capture the important aspects of robot kinematics and dynamics. These must capture the relationship between inputs and outputs so, in their simplest form, consist of a function, the kinematic mapping, between a manifold of inputs and the manipulator's configuration space.

Typically, the component bodies or segments of a robot manipulator are rigid, or at least can be treated as such for the purposes of kinematic analysis. Each can be furnished with an orthonormal coordinate frame. One component is usually designated as the base and assigned a fixed coordinate frame, though, for some problems, it may be preferable to regard all components as mobile relative to an ambient coordinate frame. Of primary interest in most problems is the motion of the end-effector - the grasping component, or component to which the operative tool is attached. Its pose (position and orientation) relative to the base can be described by a Euclidean isometry mapping its coordinate frame to that of the base.

The components of a manipulator are connected by joints of various kinds: revolute $(\mathrm{R})$, slider or prismatic $(\mathrm{P})$, screw or helical $(\mathrm{H})$, ball or spherical $(\mathrm{S})$, planar $(\mathrm{E})$. This is not an exhaustive list but these and other joints all arise from the contact of surfaces (lower kinematic pairs), or curves and points (higher kinematic pairs) in the components. The classic book of Hunt [45] provides an engineering perspective. Those joints which are subject to an input (e.g. via a servo-motor) are termed actuators, while others are passive joints. The $\mathrm{R}$ and $\mathrm{P}$-joints are simplest to engineer and their kinematic analysis is generally more straightforward. Nearly all practical manipulators use these as actuators. Some of the other joints can be synthesized by means of combinations of $\mathrm{R}$ and $\mathrm{P}$ joints. A universal (U) joint is a combination of $2 \mathrm{R}$-joints with intersecting axes while a spherical 
joint can be synthesized by $3 \mathrm{R}$-joints and both occur frequently as passive joints.

The global architecture of a manipulator (or any rigid-body mechanism) may be partially encoded combinatorially by means of a graph whose vertices are the components and edges denote a joint between components or, dually, with vertices the joints and edges the components (see, for example, [17]). Additional information is required to fully specify the robot. This information is partly topological - the nature of each joint, including its number of degrees of freedom (dofs) - and partly geometric, namely the design parameters that specify the size and relative placement of the components and joints.

A mechanism whose graph is a path is called a kinematic chain and a manipulator with this architecture is called serial. Related to serial manipulators are those whose graph is a tree, a class that includes most robot hands having fingers or other gripping mechanisms. In such manipulators, all joints are actuated.

Parallel mechanisms are those in which the end-effector, usually called the platform in this context, is connected to the base by two or more independent kinematic chains [67]. Typically, only some of the joints in each chain are actuated and if this number is 1 , the mechanism is called fully parallel, otherwise hybrid parallel. In particular, the graphs of parallel mechanisms contain cycles which impose equational constraints on the joint variables.

An important question regarding parallel mechanisms is to determine their mobility. This can be determined in the generic case from the graph using the Grübler-Kutzbach mobility formula [45] for a spatial mechanism with $n$ components, $g$ joints, the $i$ th joint having $f_{i}$ dof, $i=1, \ldots, g$ :

$$
\mathfrak{M}=6(n-g-1)+\sum_{i=1}^{g} f_{i}
$$

The mobility $\mathfrak{M}$ represents the number of inner degrees of freedom of the mechanism as a whole. An extension of this formula that takes into account symmetries has recently been derived [40]. For planar and spherical mechanisms, for example, one needs to replace 6 in (1) by 3 , the dimension of the relevant isometry subgroup. The mobility also tells us the number of joints that need to be actuated. Note however that we provide a more precise definition of mobility in the next section.

\section{Geometry of Manipulator Kinematics}

We will assume that our manipulators consist of rigid bodies connected by standard joints, operating in 3-dimensional Euclidean space. The Euclidean group of isometries, $S E(3)$ is 
isomorphic, via choice of coordinates in the moving and fixed spaces, to the semi-direct product of the proper rotation group $S O(3)$ and the additive group of translations, $\mathbb{R}^{3}$. In this form the elements of $S E(3)$ are pairs $(A, \mathbf{a}) \in S O(3) \ltimes \mathbb{R}^{3}, A$ a $3 \times 3$ orthogonal matrix with determinant +1 and $\mathbf{a}$ a 3 -vector. The group product, representing composition of isometries, is

$$
\left(A_{2}, \mathbf{a}_{2}\right) \cdot\left(A_{1}, \mathbf{a}_{1}\right)=\left(A_{2} A_{1}, A_{2} \mathbf{a}_{1}+\mathbf{a}_{2}\right) .
$$

The group acts on $\mathbb{R}^{3}$ by

$$
(A, \mathbf{a}) \cdot \mathbf{x}=A \mathbf{x}+\mathbf{a}
$$

and we can regard this is a map from the end-effector coordinates to base coordinates, describing the pose of the end-effector.

The Euclidean group is a 6-dimensional Lie group. Pure translations form a 3-dimensional normal subgroup $\mathbb{R}^{3}$, and the set of rotations about any point of $\mathbb{R}^{3}$ is also a 3-dimensional subgroup isomorphic to $S O(3)$. Two-dimensional Euclidean and spherical motion can both be regarded as special cases since the relevant isometry groups, $S E(2)$ and $S O(3)$ are (3-dimensional) subgroups of $S E(3)$. The connected Lie subgroups of $S E(3)$ have been classified by Hervé [42] (and in a different setting by Beckers et al [4]) and play an important role in mechanism theory.

Note that $S E(3)$ is also a linear algebraic group that may, for example, by defined as a real algebraic variety in $\mathbb{R}^{12}$ (representing 9 entries in a $3 \times 3$ matrix $A$ and a 3 -vector a) by means of the equations $A^{t} A=A A^{t}=I, A=\operatorname{adj} A$. Lazard [55] introduced this approach to analyze poses of the Gough-Stewart platform described below.

Elements of $S E(3)$ may be represented or parametrized in various forms [8,60,73]. For example, the rotation component can be described by

- Euler angles $(\phi, \theta, \psi)$, representing successive rotations about specific axes, so that

$$
A=\left(\begin{array}{ccc}
\cos \psi & -\sin \psi & 0 \\
\sin \psi & \cos \psi & 0 \\
0 & 0 & 1
\end{array}\right)\left(\begin{array}{ccc}
1 & 0 & 0 \\
0 & \cos \theta & -\sin \theta \\
0 & \sin \theta & \cos \theta
\end{array}\right)\left(\begin{array}{ccc}
\cos \phi & -\sin \phi & 0 \\
\sin \phi & \cos \phi & 0 \\
0 & 0 & 1
\end{array}\right)
$$

This can also be written as a product of exponentials: $\exp (\psi Z) \exp (\theta X) \exp (\phi Z)$, where $X$ and $Z$ are elements of the Lie algebra $\mathfrak{s o}(3)$.

- Rodrigues parameters, writing $A=(I-B)^{-1}(I+B), B$ skew-symmetric.

- Unit quaternions $q=c_{0}+c_{1} i+c_{2} j+c_{3} k \in \mathbb{H}$, which form a double cover of $S O(3)$ and act on $\mathbb{R}^{3}$, embedded in $\mathbb{H}$ as the pure imaginary quaternions, by $x \mapsto q x \bar{q}$ (where $\bar{q}$ denotes the quaternionic conjugate formed by negating the imaginary part of $q$ ). 
The last of these extends to representation of elements of $S E(3)$ by Clifford's dual quaternions, $q+\epsilon p$, where $q, p$ are unit quaternions and $\epsilon^{2}=0$.

An important observation is that the relative displacements permitted by the various types of joints are represented, for a choice of coordinates in each of the connected components, by subgroups of $S E(3)$, which are embedded analytic submanifolds whose dimensions are, by definition, the number of degrees of freedom of the joints. In particular, the motions defined by $\mathrm{R}, \mathrm{P}$ and $\mathrm{H}$-joints are its 1 -parameter subgroups.

The terminology for describing the kinematics of manipulators is not standardized. The following definitions are adapted from those used by the Parallel Mechanisms Information Centre [67].

Definition 2.1. Given two rigid bodies $B_{1}$ and $B_{2}$, equipped with choices of orthonormal coordinates and connected by a joint $J$, the joint space of $J$ is the submanifold of $S E(3)$ corresponding to the set of possible displacements of $B_{2}$ relative to $B_{1}$.

The joint space $P_{\mathcal{R}}$ of a robot manipulator $\mathcal{R}$ is the product of the joint spaces of all its joints, its articular space $Q_{\mathcal{R}}$ is the product of the joint spaces of its actuated joints. Its configuration space is the subset $M_{\mathcal{R}}=f^{-1}(c) \subseteq P_{\mathcal{R}}$ of attainable values for the joint variables, where $f: P_{\mathcal{R}} \rightarrow \mathbb{R}^{k}$ is a function determining the constraints.

The kinematic mapping for $\mathcal{R}$, with an identified end-effector or platform $B$ carrying an orthonormal coordinate frame, is the function $\lambda: M_{\mathcal{R}} \rightarrow S E(3)$ which to each attainable set of joint variables assigns the pose of $B$. The workspace $W_{\mathcal{R}} \subseteq S E(3)$ of $\mathcal{R}$ is the image of the kinematic mapping. In the case that $M_{\mathcal{R}}$ is a manifold, its dimension is the mobility of $\mathcal{R}$.

Given a point $\mathbf{w}$ in the end-effector, the evaluation map of $\mathbf{w}$ is the smooth function

$$
e_{w}: S E(3) \rightarrow \mathbb{R}^{3}, \quad(A, \mathbf{a}) \mapsto A \mathbf{w}+\mathbf{a}
$$

The trajectory of $\mathbf{w}$ under the kinematic mapping $\lambda$ is the smooth function

$$
\tau_{w}=e_{w} \circ \lambda: M_{\mathcal{R}} \rightarrow \mathbb{R}^{3} .
$$

Note that if one treats $S E(3)$ as an algebraic group then, for manipulators with $\mathrm{R}, \mathrm{P}$ and S-joints, $M_{\mathcal{R}}$ is a subvariety, even in the singular cases, so the mobility can be defined via the dimension of that variety. 


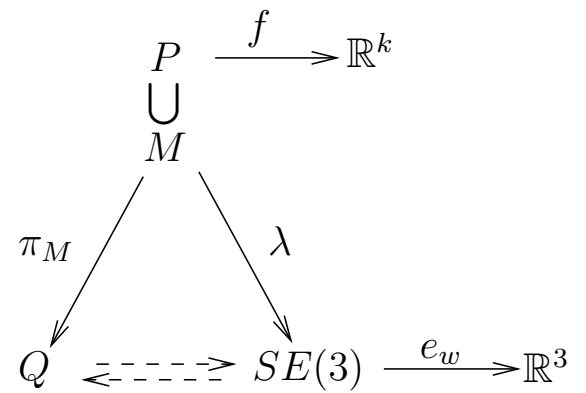

The relation between the joint, articular and configuration spaces (the subscript $\mathcal{R}$ has been suppressed) is illustrated in (2), where $\pi_{M}: M \rightarrow Q$ denotes the restriction to $M$ of the projection from the joint space to the articular space. The broken arrows between $Q$ and $S E(3)$ indicate the two fundamental relationships that underlie the control and use of manipulators. The arrow $Q \rightarrow S E(3)$ denotes the forward (or direct) kinematics of the manipulator. If it is possible to find such a function then it determines the configuration of the end-effector for a given set of joint variables. The arrow $S E(3) \rightarrow Q$ is the inverse kinematics of the manipulator, which determines the joint variables required for a given pose of the end-effector. Where distinct points in $M$ give rise to the same pose, the mechanism configurations are sometomes referred to as postures. The presence of singularities in either or both of $\lambda$ and $\pi_{M}$ obstructs the existence of global inverse or forward kinematics.

Topologically, the joint space for an $\mathrm{R}$-joint is a circle $S^{1}$ and for $\mathrm{P}$ and $\mathrm{H}$-joints an embedded real line $\mathbb{R}$. The joint space of an $\mathrm{S}$-joint is an embedded $S O(3)$. In practice, there may be physical limitations to a manipulator, restricting the effective joint space of each joint to some subset, say an interval. However to simplify the mathematical analysis we will assume the joint space to be the entire subgroup.

Hence, the joint and articular spaces of a robot manipulator are also smooth manifolds. However whether the configuration space is a manifold will depend on whether the constraint function $f$ is submersive along $f^{-1}(c)$, and that may depend on the design parameters. 


\section{Three Classes of Manipulator}

\subsection{Serial manipulators.}

For a serial manipulator, every joint is actuated so, ignoring any engineering limitations on joints, the joint space, articular space and configuration space coincide. That means the left-hand side of (2) collapses and the forward kinematics is simply the kinematic mapping. In all standard industrial manipulators the joints are either $\mathrm{R}$ or $\mathrm{P}$ so the configuration space $M$ is a product of a generalized torus $T^{r}=S^{1} \times \ldots \times S^{1}(r$ copies $)$ and a Euclidean space $\mathbb{R}^{p}$. We will see shortly that the kinematic mapping is analytic, so in order for the workspace to have non-empty interior (the end-effector has maximum freedom of translation and orientation) we require the mobility $m=r+p \geq 6$. If equality holds the manipulator is called non-redundant, while if $m>6$, it is called redundant.

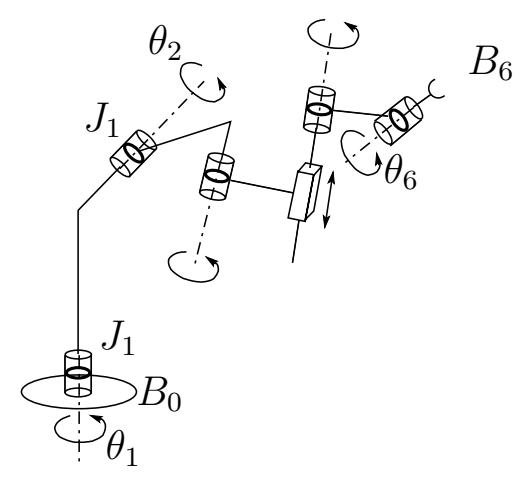

Figure 1: Serial manipulator

Associate a coordinate frame to each component and choose a 'home' configuration. Label the components in order from the base $B_{0}, \ldots, B_{m}$ and joints $J_{1}, \ldots, J_{m}$ so that joint $J_{i}$ connects $B_{i-1}$ and $B_{i}$. In the home configuration the coordinates of these components are related by an element $U_{i} \in S E(3)$. Displacements arising from $J_{i}$ form a one-parameter subgroup which can therefore be written in the form $\exp \left(\theta_{i} Y_{i}\right)$ for some $Y_{i}, i=1, \ldots, m$ in the Lie algebra $\mathfrak{s e}(3)$ (see Section 6). In particular, as was shown by Brockett [9], the kinematic mapping (= forward kinematics) for the end-effector has the form:

$$
\lambda\left(\theta_{1}, \ldots, \theta_{m}\right)=U_{1} \exp \left(\theta_{1} Y_{1}\right) U_{2} \exp \left(\theta_{2} Y_{2}\right) \ldots U_{m} \exp \left(\theta_{m} Y_{m}\right) .
$$

Judicious choice of coordinates enables one to express the matrices $Y_{i}$ in a standard form and the isometries $U_{i}$ in terms of a small number of parameters defined by the manipulator 
geometry (Denavit-Hartenberg parameters - see, for example, [19,60,63]). Alternatively, by choosing coordinate frames that coincide in the home configuration, $U_{2}=\cdots=U_{m}=1$

(where we use 1 to represent the identity in the group) the kinematic mapping can be expressed as a product of exponentials.

In practice, many industrial serial manipulators are wrist-partitioned. Orientation of the end-effector is achieved by means of a $3 \mathrm{R}$ spherical wrist - that is, the 3 axes of rotation intersect at a point, the wrist centre. Motion of the wrist relative to its centre can be represented, for example, using Euler angles. Location of the wrist centre is achieved by means of a 3-joint arm, often referred to as a regional manipulator having $p=0,1,2$ or $3 \mathrm{P}$-joints and $r=3-p \mathrm{R}$-joints. This partitioning simplifies singularity analysis as discussed in Section 4.

\subsection{Planar 4-bar mechanisms.}

The study of the kinematics of mechanisms, dating back at least to Watt's parallel motion [54] used for converting linear to rotary motion in steam engines, can be seen as a forerunner of robot kinematics. A simple but informative example of a parallel mechanism is provided by the planar 4-bar (classically referred to as the 3-bar) mechanism [34,71], of which Watt's motion is an example. The mechanism (Figure 2) consists of 4 components linked in a closed quadrilateral $A B C D$ by revolute joints. Regard the base $A D$ as fixed. $A B$ is referred to as the input bar, $B C$ as the coupler bar and $C D$ as the output bar. The design parameters are the lengths of the 4 bars $d_{i}, i=0,1,2,3$. The planar Grübler-Kutzbach formula confirms that the mobility of this mechanism is generically

$$
\mathfrak{M}=3(4-4-1)+4=1 \text {. }
$$

The joint space is a 3 -dimensional torus, parametrized by the angles $(\alpha, \beta, \gamma)$ and the constraint equations, arising from the closure of the quadrilateral, are:

$$
\begin{gathered}
d_{1} \cos \alpha+d_{2} \cos \beta+d_{3} \cos \gamma=d_{0} \\
d_{1} \sin \alpha+d_{2} \sin \beta+d_{3} \sin \gamma=0
\end{gathered}
$$

Gibson and Newstead [34] showed that the configuration space is indeed a 1-dimensional manifold (diffeomorphic to either $S^{1}$ or $S^{1} \times\{0,1\}$ ) so long as $\max \left\{d_{i}: i=0,1,2,3\right\}$ is less than the sum of the other 3 sides and the Grashof condition

$$
d_{0} \pm d_{1} \pm d_{2} \pm d_{3} \neq 0
$$




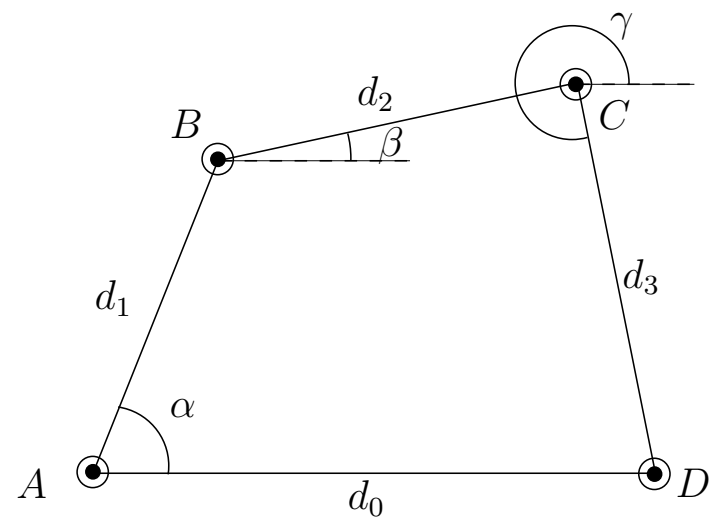

Figure 2: Planar 4-bar mechanism

is satisfied. This can be readily derived from the constraint equations (4) via the Submersion Theorem (see, for example [36]).

In classical industrial applications, the actuated joint is at $A$ and the output bar is the end-effector. The restriction to $M_{\mathcal{R}}$ of the projection of the joint space $P_{\mathcal{R}}$ onto the joint space of each joint is either onto, in which case the joint is called a crank, or not, when it is a rocker. A crank-rocker (referring to the behaviour of the input and output bars) enables conversion of rotary motion into rectilinear motion. However, from the perspective of parallel mechanisms, it makes more sense to take the coupler bar $B C$ as the end-effector (platform). The kinematic mapping in terms of $(\alpha, \beta, \gamma) \in M_{\mathcal{R}}$ is given, in one form, by

$$
\lambda(\alpha, \beta, \gamma)=\left(\left(\begin{array}{cc}
\cos \beta & -\sin \beta \\
\sin \beta & \cos \beta
\end{array}\right),\left(\begin{array}{l}
a \cos \alpha \\
a \sin \alpha
\end{array}\right)\right) \in S E(2) \cong S O(2) \ltimes \mathbb{R}^{2},
$$

but recall that the joint variables are constrained by (4).

\subsection{The Gough-Stewart Platform.}

Gough devised this famous parallel mechanism as a tyre-testing rig for Dunlop Tyres in the 1950s [39]. A similar design was later proposed by Stewart for use as the platform for a flight simulator [76]. MacCallion [59] first considered its use as a workspace manipulator. The vertices of an equilateral triangle in the base are connected pairwise to those of a similar triangle in the platform by articulated struts as in Figure 3. The 6 struts are joined to the base by universal joints and to the platform by spherical joints and each 
has a single prismatic actuator enabling the strut length to be altered (Figure 3 ). The platform is therefore a 6-UPS mechanism. This architecture is also called an octahedral hexapod. In greatest generality, the 6 struts may connect arbitrary points in the base and platform. Between these extremes are numerous architectures embodying different degrees of symmetry. In fact, the original platform properly has planar hexagonal base and platform, each with triangular symmetry. A brief history of such mechanisms can be found in Bonev [7] and a recent review of the theory is in [18].

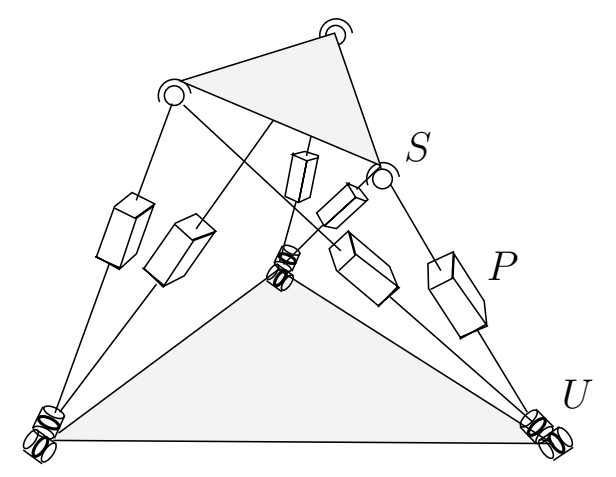

Figure 3: Gough-Stewart platform

The Grübler-Kutzbach formula predicts that such structures generically have mobility 6 (there are 14 links: $6 \times 2$ in the struts, plus base and platform; $6 \times 3=18$ joints having $6 \times 6=36$ degrees of freedom). Realizing this result in terms of Definition 2.1 is a little harder. It is simpler to replace the configuration space $M$ by $\tilde{M}$, defined to be the subset of $\left(\mathbb{R}^{3}\right)^{3} \times \mathbb{R}^{6} \cong \mathbb{R}^{15}$ representing the 3 sets of coordinates for platform vertices together with the variable strut lengths, subject to 3 independent equations fixing the distances between the vertices and 6 equations relating the coordinates to the strut lengths in terms of the base joint coordinates. The Submersion Theorem gives mobility 6 . In fact, it is clear that $\tilde{M}$ is diffeomorphic to $S E(3)$ since there is a correspondence between platform poses and feasible coordinates for the joints. The relation between $M$ and $\tilde{M}$ is less clear since it is possible that a family of joint coordinates all give rise to the same pose. (This occurs, for example of the UPS struts are replaced by SPS struts, in which case the struts are free to rotate about their axes in any pose.)

If we allow the replacement of $M$ by $\tilde{M}$ in (2), then the right-hand side of the triangle collapses and the inverse kinematics are well defined. The forward kinematics of the Gough-Stewart platform has attracted interest amongst both kinematicians and mathematicians. Methods from topology and algebraic geometry have been used to show that for a given set of actuator variables there may be up to 16 poses for the platform in the 
architecture described here (40 for the general architecture). This number depends on both the actuator variables and the design parameters and a full understanding of the way in which the number changes presents a highly technical challenge in singularity theory and topology. (For more detail, see [55,61], for example.)

\section{Instantaneous Kinematics and Singularities}

While singularities have non-local implications for the control and use of manipulators, they arise as local or instantaneous phenomena from the rank deficiency of a derivative. The diagram (2) illustrates that this may occur in a number of ways in relation to manipulators. For serial manipulators, it is the singularities of the kinematic mapping/forward kinematics and trajectories that are of interest, whereas for fully parallel manipulators it is those of the constraint function defining the configuration space and of the projection onto the articular space (inverse kinematics). The distinction between the classes of mechanisms in respect of their singularities was first recognized by Gosselin and Angeles [37] and subsequently refined by Zlatanov et al in [88,89]. Simaan and Shoham have used their ideas to analyze singularities of hybrid serial/in-parallel mechanisms [74].

The importance of singularities from an engineering perspective arises for several reasons:

(a) Loss of freedom. The derivative of the kinematic mapping or forward kinematics represents the conversion of joint velocities into generalized end-effector velocities, i.e. linear and angular velocities. This linear transformation is generally referred to as the manipulator Jacobian in the robotics literature. A drop in rank reduces the dimension of the image, representing a loss of instantaneous motion for the endeffector of one or more degrees. The proper setting for describing this is the theory of screw systems, discussed below in Section 6, which is used in many papers in the robotics literature, for example $[58,77,82]$.

(b) Workspace. When a manipulator is at a boundary point of its workspace, the manipulator is necessarily at a singular point of its kinematic mapping, though the converse is not the case. Interior components of the singular set separate regions with different numbers or topological types of inverse kinematics. These are usually associated with a change of posture in some component of the manipulator. Therefore knowledge of the manipulator singularities provides valuable information about its workspace [52].

(c) Loss of control. A variety of control systems is used for manipulators. Rate control systems require the end-effector to traverse a path at a fixed rate and therefore 
determine the required joint velocities by means of the inverse of the derivative of the (known) forward kinematics. Near a singularity, this matrix is ill-conditioned and either the control algorithm fails or the joint velocities and accelerations may become unsustainably great. Conversely, force control algorithms, well-adapted for parallel manipulators, may result in intolerable joint forces or torques near singularities of the projection onto the joint space.

(d) Mechanical advantage. Near a singular configuration, large movement of joint variables may result in small motion of the end-effector. Therefore there is mechanical advantage that may be realised as a load-bearing capacity (interesting examples in human activities are presented by Kieffer and Lenarčič [53]) or as fine control of the end-effector (an example of a telescope-focussing device is given by Carretero et al $[12,13])$. Another aspect of this is in the design of mechanisms possessing trajectories with specific singularity characteristics. In traditional 1-dof mechanisms (such as the planar 4-bar) a cusp singularity provides 'dwell' - the trajectory is close to stationary for a period of time allowing some step in a production process to be performed [44]. A higher-dimensional example is the use of a corank 3 singularity by the remote centre compliance device $[64,83,86]$ (see also Section 7 ).

The following theorems are central examples illustrating the necessity of singularity analysis for serial and parallel manipulators. The following result of Gottlieb [38], also discussed in [2], is for the forward kinematics of serial manipulators.

Theorem 4.1. For any serial manipulator with configuration space $M=T^{p} \times \mathbb{R}^{n-p}$ with $n \geq 6$, the kinematic mapping $\lambda: M \rightarrow S E(3)$ possesses singularities.

The proof is based on the observation that if there were no singularities then $\lambda$ would be a submersion, giving rise to a fibration of $\mathbb{R}^{n}$ (the universal covering space for $M$ ) over $S O(3)$. But this is ruled out on topological grounds. Moreover, it is not possible globally to avoid singularities by introducing redundancy in the manipulator. That is, for $n>6$ there is no continuous function $\theta: S E(3) \rightarrow M$ such that $\lambda(\theta(X))=X$.

There has been extensive analysis of the actual singularity configurations for industrial robot manipulators. Wang and Waldron [82] analyzed the general 6-dof serial manipulator using screw theory (see section 6) and showed that the singularity field (set of singular configurations) is independent of the joint variables $\theta_{1}$ and $\theta_{6}$. Litvin et al $[57,58]$ explicitly examined $6 \mathrm{R}$ manipulators and showed that there were three sets of singularities relating to different configurations, subsequently dubbed wrist, elbow and shoulder singularities. Stanišić and Engelberth [75] looked at wrist-partitioned manipulators, where 
the Jacobian determinant factors into one component for the wrist and one for the arm subassembly. They showed that there are surfaces in $\mathbb{R}^{3}$, dependent on the manipulator's configuration, such that when the wrist centre lies on them then the manipulator is in a singular configuration. The surfaces themselves are determined by the associated screw system of the arm subassembly in its current configuration, described in further detail in Sections 6 and 7 .

In $[78,79]$, Tchoń and Muszynski sought to characterize serial manipulator singularities by finding normal forms with respect to $\mathcal{A}$-equivalence (right-left equivalence). They showed that among corank 1 singularities (i.e. away from intersections of the singular surfaces), the elbow and shoulder singularities are folds and hence stable, but the wrist singularities have infinite $\mathcal{A}$-codimension ('differential degree' in their terminology).

In relation to fully parallel manipulators of the Gough-Stewart kind, Merlet [61] showed the following:

Theorem 4.2. The inverse kinematics of a 6-UPS parallel mechanism are singular if and only if the lines spanned by the 6 struts are linearly dependent.

This follows because the rows of the inverse Jacobian can be shown to be the Plücker line coordinates of the struts. Again this is closely linked to the theory of screw systems.

It is worth noting here that for parallel manipulators, the full joint space is important for singularity analysis, since there may be configurations for which platform motion is possible because of passive joint velocities. This was originally observed by di Gregorio and Parenti-Castelli [20] and has been the subject of further exploration, under the terminology constraint singularities by Zlatonov et al [87].

\section{Genericity and Transversality Theorems}

\subsection{One-genericity}

A general theory for kinematic mappings $\lambda$ can be set up by considering spaces of smooth (or analytic) mappings $C^{\infty}(M, S E(3))$ where $M$ is the configuration space of a manipulator or, locally, germs of such mappings. The Whitney Immersion Theorem (see, for example, [36]) assures us that for manipulators with up to 3 dof, there is an open and dense set of mappings that are immersions (and if $M$ is compact, then this is true for 1-1 immersions). In practice, we are frequently interested in cases where $\operatorname{dim} M \geq 6$, and for dimension 4 and upwards singularities will occur stably. 
Several authors have sought to identify generic properties for kinematic mappings - that is, properties possessed by a suitably large subset, say open and dense, or at least residual. A starting point is usually to identify relevant submanifolds of a jet bundle $J^{k}(M, S E(3))$ (or multi-jet bundle) and require transversality to these for the associated jet extension. Then the Thom Transversality Theorem [36] guarantees genericity.

Pai and Leu [65] adopt this approach, using the stratification of the 1-jet bundle by corank, that is $\Sigma^{r}=\left\{\sigma \in J^{1}(M, S E(3))\right.$ : corank $\left.\sigma=r\right\}$. A kinematic mapping transverse to this stratification is called 1 -generic. They analyze the standard architectures for serial manipulators from this perspective, distinguishing the orientation singularities of the wrist from the translational singularities of the regional manipulator, by composing the kinematic mapping with projection onto its components. In the case of translations, this projection is coordinate dependent and amounts to analyzing the trajectory of the wrist centre. The singular point set in the joint space is always invariant under rotation about the first axis. Hence, in the generic case where $\Sigma^{r} \lambda=\left(j^{1} \lambda\right)^{-1}\left(\Sigma^{r}\right)$ can only be non-empty for $r=0,1$, the singular point set can be identified with a union of circles in the 2 -torus corresponding to $\theta_{2}, \theta_{3}$ in equation (3). This led Burdick [11] to propose a classification of $3 \mathrm{R}$ regional manipulators with generic kinematic mapping based on the homotopy class(es) of the components of $\Sigma^{1} \lambda$.

Subsequent work by Wenger et al $[1,84,85]$ resolved some conjectures of Burdick concerning 3R manipulators. In particular, they showed that the presence of a cusp singularity, that is $\Sigma^{1,1}$ in the Thom-Boardman classification, is a necessary and sufficient condition for the existence of a path in the configuration space realizing a change of posture without encountering a singularity. This is an interesting result in the area of singularity avoidance, which is concerned with the topology of the singularity field.

\subsection{Trajectory singularities and a transversality theorem}

A deeper approach was pursued by Gibson et al. They sought to examine the relation between the kinematic mapping $\lambda=(A, \mathbf{a})$ and its family of trajectories

$$
\tau_{\lambda}=e_{w} \circ \lambda: M \times \mathbb{R}^{p} \rightarrow \mathbb{R}^{p}, \quad(x, w) \mapsto A(x) \mathbf{w}+\mathbf{a}(x)
$$

for planar $(p=2)$ and spatial $(p=3)$ kinematics. The key result is the following theorem of Gibson and Hobbs [28]. For integers $k, r \geq 1$, there is a multijet extension

$$
{ }_{r} j_{1}^{k} \tau_{\lambda}: M^{(r)} \times \mathbb{R}^{p} \rightarrow{ }_{r} J^{k}\left(M, \mathbb{R}^{p}\right)
$$

where ${ }_{1}$ means take jets w.r.t. first component only. This map assigns the $k$-jets of the $p$-parameter family of trajectories to a set of $r$ distinct configurations in $M$. 
Theorem 5.1. Given a finite stratification $\mathcal{S}$ of the multijet bundle ${ }_{r} J^{k}\left(M, \mathbb{R}^{p}\right)$, the set of $\lambda \in C^{\infty}(M, S E(p))$ such that ${ }_{r} j_{1}^{k} \tau_{\lambda}$ is transverse to $\mathcal{S}$ is residual.

The original proof followed that of Wall [81] for singularities of projections of generic immersions; a simpler proof, using the fact that the evaluation map is a submersion, follows from a theorem of Montaldi [62] on composite maps. Note that this, in some sense, subsumes Pai and Burdick's approach for regional manipulators, since that concerns the wrist-centre trajectory, by taking $r=k=1$ and $\mathcal{S}$ the corank stratification. The theorem underpins a programme, described in [26], for exploring singularities of trajectories.

- Classify $\mathcal{A}$-types of multigerm singularities up to relevant codimension. Subject to amalgamating orbits with moduli, this typically provides an $\mathcal{A}$-invariant finite stratification of the multijet bundles.

- Transversality imposes constraints on codimension of strata that can be encountered. Gibson and Hobbs [28] show that the requirement for a non-stable multigerm with $\mathcal{A}$-modality $m$ to occur transversely is that its $\mathcal{A}_{e}$-codimension is $\leq \operatorname{dim} M+m$.

- Versal unfoldings of these singularity types give local models for the bifurcation sets in $\mathbb{R}^{p}$.

In a sequence of papers [25,27-31,33,41,43], Gibson and co-workers filled out details of this programme for planar and spatial motions up to 3-dof, in the process generating new lists of (multi-)singularities of maps between spaces of dimension up to 3. It was shown in [25] that the stable singularity type of the kinematic mapping germ itself imposes restrictions on the singularity type of its trajectory germs. However the $\mathcal{A}$-classification of mapgerms becomes computationally harder as the number of degrees of freedom increase. For example, there are more than 50 classes up to $\mathcal{A}_{e}$-codimension 3 for 3 -dof spatial motions [41].

\subsection{Problems with genericity}

There is a fundamental difficulty with the genericity approach. The idea of genericity is to identify properties of mappings that are typical among all such mappings. The space $C^{\infty}(M, S E(3))$ is infinite-dimensional. However in robotics one is almost always concerned with a specific class of manipulators defined by a finite number of design parameters. It is by no means sure that this finite-dimensional space will lie in such a way that a given property that is generic in $C^{\infty}(M, S E(3))$ will remain so on restriction to the subset. One 
can use the Elementary Transversality Theorem [36] in this setting but that is likely to require explicit calculation of transversality, stratum by stratum.

For serial manipulators, the design parameters can be chosen to be Denavit-Hartenberg parameters. Pai and Leu showed explicitly that within this parameter space, all the standard regional manipulator architectures (i.e. with a combination of $3 \mathrm{R}$ or $\mathrm{P}$ joints) and orientation (wrist) architectures have an open and dense set - the complement of an analytic function in the parameters - of 1-generic mappings.

However even in one of the simplest cases involving parallelism, coupler curves of planar 4bar mechanisms, it has not yet been established that the restricted version of the GibsonHobbs Transversality Theorem holds, the remaining obstruction being the monogerm stratum of $A_{4}$ singularities (ramphoid cusps) [14].

A second drawback is that these approaches do not explicitly take into account the structure of $S E(3)$ or its tangent spaces. In a singular configuration we are interested not only in the dimension of the image of the derivative but also in how it lies with respect to that structure.

Finally, it is important to note that from an engineering perspective it is often advantageous to use special, that is non-generic, architectures in order to achieve desirable motion characteristics. One example is the emphasis on wrist-partitioned serial manipulators with only $\mathrm{R}$ and $\mathrm{P}$ joints. Another example is the interest in so-called over-constrained structures, where the mobility exceeds that predicted by the Grübler-Kutzbach formula. A well-known example is the Bennett mechanism [5,6], a closed 4-link kinematic chain with 1 dof, though the formula predicts that spatial closed chains with up to 6 links should be rigid. The study of singularities is relevant to over-constrained mechanisms because their motion can be regarded as a motion contained entirely within the subset of singular configurations of an associated open chain or serial mechanism (realised by unlinking one of the joints). This approach to closed mechanisms is exploited, for example, by Lerbet and Hao [56].

\section{Screw Systems}

Theorems 4.1 and 4.2 indicate the importance of the tangent spaces to the Lie group $S E(3)$ as either the range or domain of the relevant derivatives. The essential structure is that of the Lie algebra $\mathfrak{s e}(3)$, which can be variously identified as the tangent space to $S E(3)$ at the identity, the space of one-parameter subgroups of $S E(3)$ or the space of Killing vector fields on $\mathbb{R}^{3}$ (see, for example, [72]). It inherits from the group structure described in Section 1 the structure of a semi-direct product of the Lie algebras $\mathfrak{s o}(3)$ of 
the rotation group and $\mathfrak{t}(3)$ of the translation group. Thus, elements may be represented by a pair $(B, \mathbf{v}) \in \mathfrak{s o}(3) \ltimes \mathfrak{t}(3)$ where $B$ is a skew-symmetric $3 \times 3$ matrix and $\mathbf{v}$ a 3 -vector.

If $B$ has the form

$$
\left(\begin{array}{ccc}
0 & -u_{3} & u_{2} \\
u_{3} & 0 & -u_{1} \\
-u_{2} & u_{1} & 0
\end{array}\right)
$$

then it can be identified with the vector $\mathbf{u}=\left(u_{1}, u_{2}, u_{3}\right)^{t}$ that (if non-zero) spans its kernel. Thus elements of $\mathfrak{s e}(3)$ can be represented by 6 -vectors $(\mathbf{u}, \mathbf{v})$. The orbits of the associated Killing vector fields are illustrated in Figure 4.

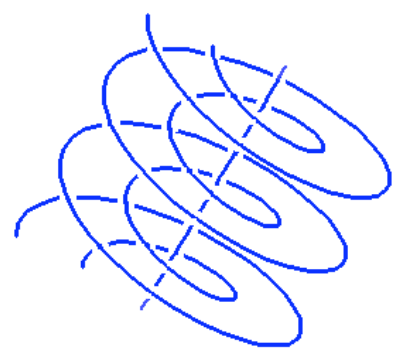

(a) $\mathbf{u} \neq 0$

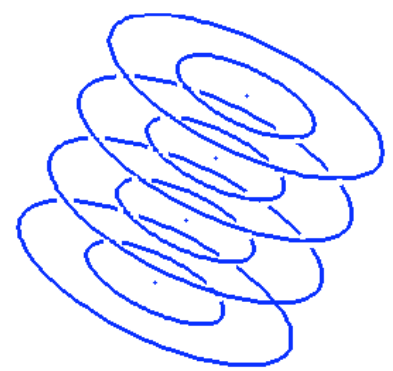

(b) $\mathbf{u}=0, \mathbf{v} \neq 0$

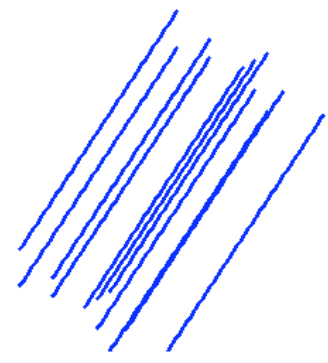

(ii) $\mathbf{u} . \mathbf{v}=0$

(i) $\mathbf{u} . \mathbf{v} \neq 0$

Figure 4: Infinitesimal motions of $\mathfrak{s e}(3)$

Following ideas in $[21,22]$, the following local equivalence was defined in [23], where it is assumed that coordinates are chosen so that at the configuration $x \in M, \lambda(x)=1$ (the group identity).

Definition 6.1. Two kinematic mapping germs $\lambda_{i}: M, x \rightarrow S E(n), 1, i=1,2$ are $\mathcal{I}$ equivalent if there exists a germ of a diffeomorphism $\phi: M, x \rightarrow M, x$ and an element $g \in S E(n)$ such that

$$
\lambda_{2}=g^{-1} \cdot\left(\lambda_{1} \circ \phi\right) \cdot g
$$

First-order invariants for $\mathcal{I}$-equivalence arise from the adjoint action of $S E(3)$ on $\mathfrak{s e}(3)$. The Lie algebra has non-trivial radical (maximal solvable ideal) $1 \ltimes \mathfrak{t}(3)$ so is not semisimple. In particular its Killing form is degenerate and the polynomial invariant theory for reductive algebras does not apply. Donelan and Gibson [23] determined generators for the ring of invariant polynomials for the adjoint action of $S E(n)$ and in particular: 
Theorem 6.2. The ring of invariant polynomials of the adjoint action of $S E(3)$ on $\mathfrak{s e}(3)$ is generated by the Killing form $\langle\mathbf{u}, \mathbf{u}\rangle$ and the Klein form $\langle\mathbf{u}, \mathbf{v}\rangle$.

This is a special case of the theorem of Panyushev for semi-direct products $\mathfrak{g} \ltimes V$ with $\mathfrak{g}$ reductive [66].

The effect of $\phi$ in Definition 6.1 is that we are only interested in the subspace $T_{x} \lambda(M) \subseteq$ $\mathfrak{s e}(3)$, which can be regarded as an element of the Grassmannian of subspaces of dimension $k=\operatorname{rank} T_{x} \lambda$. Elements of the projective space $P \mathfrak{s e}(3)$ are called screws. Since the invariants in Theorem 6.2 are both quadratic, their ratio $h=\langle\mathbf{u}, \mathbf{v}\rangle /\langle\mathbf{u}, \mathbf{u}\rangle$ is a projective invariant, called the pitch of the screw, and indeed it measures the displacement parallel to the axis during one revolution, as in Figure 4 (a)(i). In the case $\mathbf{u}=0$, set $h=\infty$.

Since the adjoint action is linear it induces an action on the Grassmannians. The theory of screws and screw systems was first developed extensively by Ball [3] and later revived by Hunt [45]. Hunt put forward a classification scheme based on geometric and engineering intuition. He noted that in engineering it was almost always the special systems that were of interest. A mathematical foundation for the classification was provided by Gibson and Hunt [32]. The principles underlying the classification, which is $\mathcal{I}$-invariant, are the following [23].

(a) $P \mathfrak{s e}(3)$ is partitioned by the pencil of quadric hypersurfaces of constant pitch:

$$
Q_{h}(\mathbf{u}, \mathbf{v})=\langle\mathbf{u}, \mathbf{v}\rangle-h\langle\mathbf{u}, \mathbf{u}\rangle=0, \quad h \in \mathbb{R} \cup\{\infty\} .
$$

where we use $Q_{h}$ to denote both the quadratic form and the associated hypersurface. For $h \neq \infty, Q_{\infty} \subset Q_{h}$ so properly we should use $\tilde{Q}_{h}=Q_{h}-Q_{\infty}$. For $h \neq \infty$ these quadrics have two rulings: by the $\alpha$-planes, corresponding to the screws of pitch $h$ whose axes pass through a given point, and by the $\beta$-planes, corresponding to those whose axes lie in a given plane. $Q_{0}$ corresponds to the classical Klein quadric, representing the set of lines in projective 3-space, in terms of Plücker line coordinates, which the screw coordinates generalize. It plays a special role in that its axis (see Figure 4 (a)(ii)) consists of instantaneously stationary points and these are the only screws having such points.

(b) Classify screw systems of a given dimension by how they meet this pencil of quadrics:

(a) Type I systems do not lie wholly in a pitch quadric and type II do.

(b) Subtypes A, B, C, D are classified according to the dimension of their intersection with $Q_{\infty}$. 
(c) Type I subclasses are further subdivided by the projective type of the pencil of intersections. For example 3-systems intersect the pitch quadrics in a pencil of real conics. Subtype IA systems can be distinguished by whether their three principle pitches, corresponding to singular conics in the pencil, are distinct $\left(\mathrm{IA}_{1}\right)$ or whether two coincide $\left(\mathrm{IA}_{2}\right)$.

(d) Further refinement is provided by the signs of the moduli such as principal pitches [24], e.g. type $\mathrm{IA}_{1}^{+0-}$ denotes the subclass with principal pitches $h_{\alpha}>0$, $h_{\beta}=0$ and $h_{\gamma}<0$.

(c) Each $Q_{h}$ derives from an associated bilinear form which gives rise to a polarity on the set of screws: $Q_{h}\left(\$_{1}, \$_{2}\right)=0$. Polarity with respect to $Q_{0}$, called reciprocity, has a particular physical significance in that screws can be used to represent both infinitesimal motion and generalized force (force + torque). Reciprocity indicates that a generalized force on a screw $\$_{1}$ produces zero rate of work on a body free to move on screw $\$_{2}$. The set of screws $S^{\perp}$ reciprocal to a $k$-system $S$ is itself a $(6-k)$-system, so one can deduce a classification of $(6-k)$-systems from that for $k$-systems, $k=1,2$.

In [24], it is shown that all the classes described above are submanifolds in the relevant Grassmannian and their adjacency diagrams are established. In particular:

Theorem 6.3. The Hunt-Gibson classification of screw systems and its refinement form Whitney regular stratifications of the relevant Grassmannians.

The stratifications can be translated across the jet bundle $J^{1}(M, S E(3))$, within motion germs of each rank, to give a regular stratification. It follows from the transversality theorem for stratified sets [35] that for a residual set in $C^{\infty}(M, S E(3))$ the 1-jet extension is transverse to the stratification. In particular, generically, we only encounter screw systems up to codimension $\operatorname{dim} M$ in the Grassmannian. For example, for $\operatorname{dim} M=3$, type $\mathrm{IA}_{1}^{+0-}$ has codimension 1 so one would expect to find a surface in $m$ along which the screw system is of this type; but IIA ${ }^{0}$, which is an $\alpha$-plane in $Q_{0}$, has codimension 6 so one does not expect to encounter this 3 -system generically.

It should be noted that there exist other classifications, though essentially equivalent, such as that of Rico Martínez and Duffy $[69,70]$ and there does not, at this stage, appear to be an accepted standard.

The relevance of screw systems to the study of kinematic singularities is obvious. For example, a 6-link serial manipulator is in a singular configuration precisely when the screws defined by its joints span a screw system of dimension $\leq 5$. Karger has written 
several papers [48-50] exploring the singularities of serial manipulators. Starting from the product of exponentials formula (3), he highlights the connection with closed loops and the significance of not only the screw system itself but also the Lie algebra it generates.

\section{Instantaneous Singular Sets and Applications}

The Transversality Theorem 5.1 indicates a connection between a kinematic mapping $\lambda$ and singularities of the associated family of trajectories $\tau_{\lambda}$. However, the equivalence relations do not preserve much of the rigid geometry that is an explicit feature of kinematic mappings. On the other hand, $\mathcal{I}$-equivalence does preserve this geometry so it is natural to study trajectory singularities in the context of screw theory.

Definition 7.1. Given the germ of a motion $\lambda: M, x \rightarrow S E(n), 1$, its instantaneous singular set (ISS) at $x$ is

$$
I_{\lambda, x}=\left\{\mathbf{w} \in \mathbb{R}^{n}: \tau_{\lambda, w} \text { singular at } x\right\}
$$

where $\tau_{\lambda, w}=e_{w} \circ \lambda$.

For example, for a $3 \mathrm{R}$ regional manipulator, the ISS in a given configuration is precisely the union of singular surfaces identified by Stanišić and Engelberth [75]. Indeed, they characterize these surfaces in terms of the principal pitches of the associated 3-system. Their classification can be derived from the following [15] which applies to screw systems of any dimension.

Theorem 7.2. Let $\lambda: M, x \rightarrow S E(3), 1$ be a kinematic mapping germ with rank $d$ and let $S$ be the associated screw system. For a point $\mathbf{w} \in \mathbb{R}^{3}$ let $A_{w}$ be the $\alpha$-plane in the Klein quadric $Q_{0}$ representing the bundle of lines through $\mathbf{w}$. Then $\mathbf{w} \in I_{\lambda, x}$ if and only if $S \cap A_{w}$ has projective dimension $\geq \max (0, d-3)$.

This follows by applying the Chain Rule to $\tau_{\lambda, w}$ :

$$
\begin{aligned}
\mathbf{w} \in I_{\lambda, x} & \Longleftrightarrow \operatorname{rank} T_{x} \tau_{\lambda, w}<\min \{d, 3\} \\
& \Longleftrightarrow \operatorname{dim}\left(\operatorname{im} T_{x} \lambda \cap \operatorname{ker} T_{1} e_{\mathbf{w}}\right)>\max \{0, d-3\}
\end{aligned}
$$

and noting that the relevant subspaces correspond to $S$ and $A_{w}$ on projectivization. An important corollary is that the ISS is the union of all the lines corresponding to points of $S \cap A_{w}$ satisfying the condition of the theorem. Also, the affine dimension of the intersection in (5) is precisely the corank of the singularity of the trajectory of $\mathbf{w}$. Moreover, 
it was also shown that the ISS of a screw system is identical to the ISS of its reciprocal system.

It is a straightforward exercise to describe the ISS associated to a given class of screw systems. In the simplest cases of a $3-$ system, $\mathrm{IA}_{1}^{+--}$, for example, the ISS is an elliptic single-sheeted hyperboloid, and each point on it has a singularity of corank 1 . For type $\mathrm{IA}_{1}^{+0-}$, where the intersection with $Q_{0}$ is a line pair (singular conic) rather than a nonsingular conic, the ISS is an intersecting pair of planes and on the line of intersection there are two distinguished points which have corank 2 singularities. For type IIA ${ }^{0}$, corresponding to an $\alpha$-plane of lines through a given point in $\mathbb{R}^{3}$, the whole space is singular but the given point has a corank 3 singularity. From the point of view of singularity theory, such a singularity is highly non-generic.

As a final application, we consider a family of parallel manipulators: those for which three points on the platform are constrained to lie on three given surfaces (not necessarily distinct), as in Figure 5. There are connections with the research of Pottmann and Ravani [68] on the singularities of motions where the constraint is that one surface (e.g. a milling head) is required to be in contact with another. However they use only the line geometry relevant to screws of zero pitch rather than full screw systems.
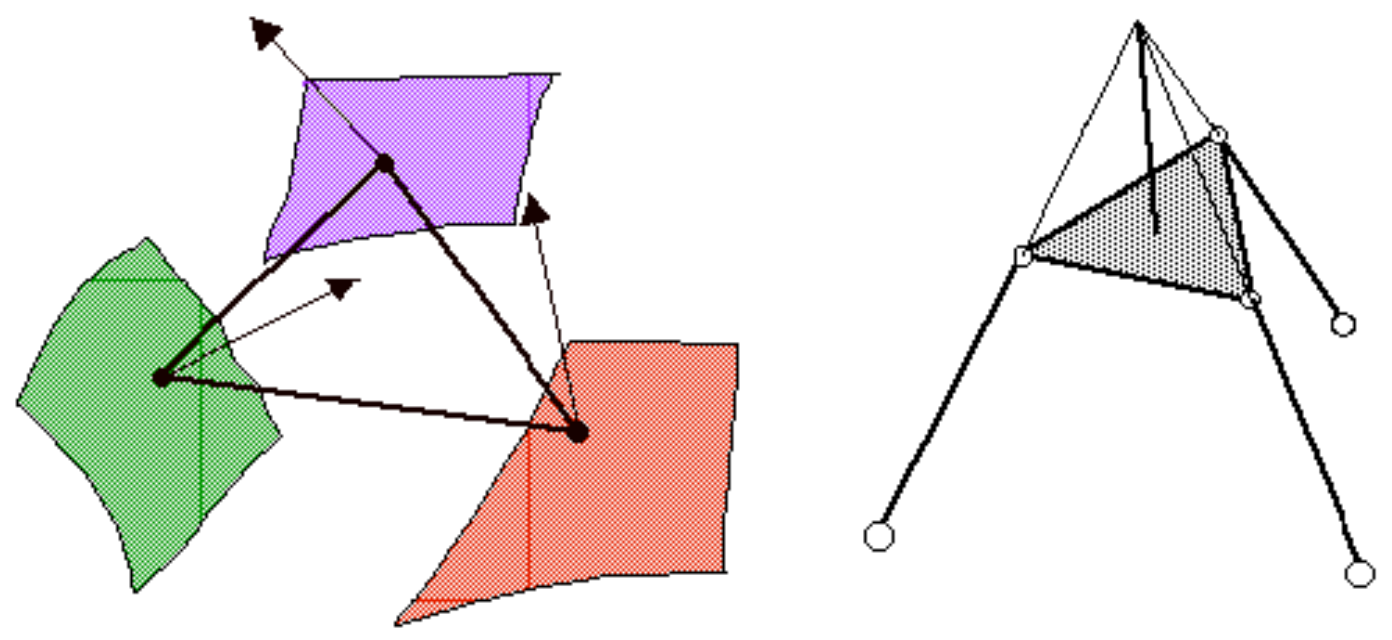

Figure 5: Three-point constrained parallel manipulator and RCC

Examples are:

- the Darboux motion, described in [8], where three points in a body are constrained to lie in three planes in general position 
- the telescope-focussing mechanism of Carretero et al $[12,13]$, where three points on the telescope mirror lie on 3 mutually intersecting planes

- a simplified model of the remote centre compliance device [86], illustrated, in which the contact points are constrained to lie on symmetrically placed, congruent spheres.

It was shown in $[14,16]$ that the screw system in any given configuration is determined by the surface normal lines at the contact points; thought of as screws of pitch 0, they span the reciprocal system. It follows that the normal lines lie in the ISS of the reciprocal system and hence the screw system of the motion itself. This enables us to deduce the screw type from the geometric configuration of the lines. For example, if the normal lines are mutually skew and their directions span $\mathbb{R}^{3}$ then the screw system type is $\mathrm{IA}_{1}^{++-}$or $\mathrm{IA}_{1}^{+--}$. If, however, the normals have independent directions but intersect in a finite point then the type is $\operatorname{IIA}^{0}$.

Among all 3 -dof motions one does not encounter type IIA $^{0}$ transversely since it forms a stratum of codimension 6 . Moreover it is associated to the existence of a trajectory of corank 3 and hence $\mathcal{A}$-codimension at least 9 (sitting within a 3 -parameter family of trajectories). However, among 3-point constrained parallel motions we have the following [16].

Theorem 7.3. Suppose a 3-point motion has a type IIA $A^{0}$ screw system. So long as a simple transversality condition is satisfied, then there is an open neighbourhood of contact triangles (and indeed of contact surfaces), containing the given motion, for which the corresponding motion also has a type IIA ${ }^{0}$ screw system.

For a Darboux motion with acute contact triangle and for the simplified RCC, there exist configurations at which the screw system is type IIA $^{0}$ and the conditions of Theorem 7.3 are satisfied. Hence these can be regarded as stable occurrences, meaning that the class of surface--constrained motions is not generic in the sense of Gibson and Hobbs.

A similar theorem holds also for type IIB $^{0} 3$-systems, which also form a class of codimension 6. This includes the telescope-focussing device. However in that case the transversality condition fails, meaning that the singular phenomenon being utilized is sensitive to the design parameters of the mechanism.

\section{Conclusion}

Singularities are of great interest and importance in robot manipulator design and control. A number of attempts have been made to apply the methods and perspective of 
singularity theory. This has resulted in the discovery of some powerful general kinematic theorems and, in some cases, a better understanding of the singularities of specific classes of manipulators. However there is a tension between the engineering and mathematical approaches. Many practical manipulators have special geometries that render statements about generic situations inapplicable. In practice, one often focusses on the non-generic cases, though here singularity theory can provide the right conceptual framework and language and may provide standard local models of the bifurcation set within the manipulator class.

There are several directions for future research in robot manipulator singularities that are likely to be fruitful. These include:

- Further exploration of specific finite-dimensional classes of manipulators with a view to finding transversality theorems in the spirit of, for example, [10].

- A more detailed understanding of the singularities of serial robot manipulators, where the product of exponentials representation of the kinematic mapping highlights the important interaction with the Lie algebra structure of the screw space.

- Application of algebraic and semi-algebraic singularity theory to the study of parallel manipulators where, frequently, the relevant constraints and mappings can be written in polynomial form. This is linked to generalizations of Kempe's Theorem [51] that (arbitrary large sections of) every plane algebraic curve can be generated as the output curve of a planar mechanism.

- The identification of higher order invariants of the adjoint action. For time dependent motion these were developed by Veldkamp $[8,80]$. These are also important for understanding the differential-geometric properties of manipulator motions and their trajectories.

- Exploration of the role of symmetry on manipulator singularities. Frequently, the presence of symmetries can give rise to unexpected singularity types. For example, most of the known over-constrained closed loop mechanisms possess symmetries and $\mathbb{Z}_{2}$-symmetries arise where mechanisms have up-down poses arising from consecutive revolute joints.

- Extension of existing results on singularity avoidance through determination of the topology of the sets of singularities and how these bifurcate under change of design parameters. 
- Links to control theory and Lagrangian singularities for the dynamics of manipulators. It is already known that there are subtle links between the geometry of mechanisms and the theory of caustics [10] so it is not unreasonable to expect that there are links between symplectic geometry and the singularities of manipulators.

\section{References}

[1] Baili, M., Wenger, P. and Chablat, D., Classification of One Family of 3R Positioning Manipulators, Proc. 11th Int. Conf. on Advanced Robotics, Coimbra, Portugal, 2003

[2] Baker, D. R., Some Topological Problems in Robotics, The Mathematical Intelligencer, 12 (1990) 66-76

[3] Ball, R. S., The Theory of Screws, Cambridge University Press, Cambridge, 1900

[4] Beckers, J., Patera, J., Perroud, M. and Winternitz, P., Subgroups of the Euclidean Group and Symmetry Breaking in Nonrelativistic Quantum Mechanics, J. Math. Phys., 18 (1977) 72-83

[5] Bennett, G. T., A New Mechanism, Engineering, 76 (1903) 777-778

[6] Bennett, G. T., The Skew Isogram Mechanism, Proc. London Math. Soc. (2nd series), 13 (1913) 151-173.

[7] Bonev, I., The True Origins of Parallel Robots, ParalleMIC Reviews, 7 (2003) http://www.parallemic.org/Reviews/Review007.html

[8] Bottema, O. and Roth, B., Theoretical Kinematics, Dover Publications, New York, 1990

[9] Brockett, R., Robotic Manipulators and the Product of Exponentials Formula, in Proc. Mathematical Theory of Networks and Systems, Beer-Sheva, Israel, ed. P Fuhrman, (1984) 120-129

[10] Bruce, J. W., Giblin, P. J. and Gibson, C. G., On Caustics by Reflexion, Topology, 21 (1982) 179-199

[11] Burdick, J. W., A Classification of 3R Regional Manipulator Singularities and Geometries, Mechanism and Machine Theory, 30 (1995) 71-89 
[12] Carretero, J. A., Nahon, M., Buckham, B. and Gosselin, C. M., Kinematic Analysis of a Three-DoF Parallel Mechanism for Telescope Applications, Proc. ASME Design Engineering Technical Conf., Sacramento, ASME, 1997

[13] Carretero, J. A., Podhorodeski, R. P. and Nahon, M., Architecture Optimization of a Three-DoF Parallel Mechanism , Proc. ASME Design Engineering Technical Conf., Atlanta ASME, 1998

[14] Cocke, M. W., Natural Constraints on Euclidean Motions, PhD Thesis, Department of Mathematical Sciences, University of Liverpool, 1998

[15] Cocke, M. W., Donelan, P.S. and Gibson, C. G., Instantaneous Singular Sets Associated to Spatial Motions, in Real and Complex Singularities, São Carlos, 1998, eds. F. Tari and J. W. Bruce, Res. Notes Math., 412, Chapman and Hall/CRC Press, Boca Raton, (2000) 147-163

[16] Cocke, M. W., Donelan, P.S. and Gibson, C. G., Trajectory Singularities for a Class of Parallel Mechanisms, presented at Real and Complex Singularities, Luminy, France, 2004

[17] Connolly, R. and Demaine, E. D., Geometry and Topology of Polygonal Linkages, Handbook of Discrete and Computational Geometry, 2nd ed., CRC Press, Boca Raton, (2004) 197-218

[18] Dasgupta, B. and Mruthyunjaya, T. S., The Stewart Platform: a Review, Mechanism and Machine Theory, 35 (2000) 15-40

[19] Denavit, J. and Hartenberg, R. S., A Kinematic Notation for Lower Pair Mechanisms based on Matrices, ASME J. Applied Mechanics, 22 (1955) 215-221

[20] Di Gregorio, R. and Parenti-Castelli, V., Mobility Analysis of the 3-UPU Parallel Mechanism Assembled for a Pure Translational Motion, Proc. IEEE/ASME Int. Conf. on Advanced Intelligent Mechatronics, Atlanta, Georgia, (1999) 520-525

[21] Donelan, P. S., Generic Properties of Euclidean Kinematics, Acta Applicandae Mathematicae, 12 (1988) 265-286

[22] Donelan, P. S., On the Geometry of Planar Motions, Quarterly J. Math. Oxford, 44 (1993) 165-184

[23] Donelan, P. S. and Gibson, C. G., First-Order Invariants of Euclidean Motions, Acta Applicandae Mathematicae 24 (1991) 233-251 
[24] Donelan, P. S. and Gibson, C. G., On the Hierarchy of Screw Systems, Acta Applicandae Mathematicae, 32 (1993) 267-296

[25] Donelan, P. S., Gibson, C. G. and Hawes, W., Trajectory Singularities of General Planar Motions, Proc. Royal Soc. Edinburgh, 129A, (1999) 37-55

[26] Gibson, C. G., Kinematic Singularities - A New Mathematical Tool, Proc. 3rd Int. Workshop on Advances in Robot Kinematics, Ferrara, Italy, (1992) 209-215.

[27] Gibson, C. G., Hawes, W. H. and Hobbs, C. A., Local Pictures for General TwoParameter Motions of the Plane, Advances in Robot Kinematics and Computational Geometry, Kluwer Academic Publishers, (1994) 49-58.

[28] Gibson, C. G. and Hobbs, C. A., Simple Singularities of Space Curves, Math. Proc. Camb. Phil. Soc., 113 (1992) 297-310

[29] Gibson, C. G. and Hobbs, C. A., Local Models for General One-Parameter Motions of the Plane and Space, Proc. Royal Soc. Edinburgh, 125A (1995) 639-656

[30] Gibson, C. G. and Hobbs, C. A., Singularity and Bifurcation for General TwoDimensional Planar Motions, New Zealand J. Math., 25 (1996) 141-163

[31] Gibson, C. G., Hobbs, C. A. and Marar, W. L., On Versal Unfoldings of Singularities for General Two-Dimensional Spatial Motions, Acta Applicandae Mathematicae, 47 (1996) 221-242

[32] Gibson, C. G. and Hunt, K. H., Geometry of Screw Systems I \& II, Mechanism and Machine Theory, 25 (1990) 1-27

[33] Gibson, C. G., Marsh, D. and Xiang, Y., Singular Aspects of Generic Planar Motions with Two Degrees of Freedom, Int. J. Robotics Research, 17 (1998) 1068-1080

[34] Gibson, C. G. and Newstead, P. E., On the Geometry of the Planar 4-Bar Mechanism, Acta Applicandae Mathematicae, 7 (1986) 113-135

[35] Gibson, C. G., Wirthmüller, K., Du Plessis, A. A. and Looijenga, E., Topological Stability of Smooth Mappings, Lecture Notes in Mathematics 552, Springer Verlag, Berlin, 1976

[36] Golubitsky M. and Guillemin V. Stable Mappings and Their Singularities, Springer Verlag, New York, 1973 
[37] Gosselin, C. and Angeles, J., Singularity Analysis of Closed-Loop Kinematic Chains, IEEE Trans. Robotics and Automation, 6 (1990) 281-290

[38] Gottlieb, D. H., Robots and Fibre Bundles, Bull. Soc. Math. Belg., 38 (1986) 219-223

[39] Gough, V. E. and Whitehall, S. G., Universal Tyre test Machine, Proc. 9th Int. Technical Congress FISITA, (1962) 117-137

[40] Guest, S. D. and Fowler, P. W., A Symmetry-Extended Mobility Rule, Mechanism and Machine Theory, 40 (2005) 1002-1014

[41] Hawes, W., Multi-Dimensional Motions of the Plane and Space, Ph. D. Thesis, University of Liverpool, 1995

[42] Hervé, J. M., Analyse Structurelle des Mécanismes par Groupe des Déplacements, Mechanism and Machine Theory, 13 (1978) 437-450

[43] Hobbs, C. A., Kinematic Singularities of Low Dimension, Ph. D. Thesis, University of Liverpool, 1993

[44] Hobbs C. A., Singularities of Mechanisms with One Degree of Freedom, unpublished

[45] Hunt, K. H. Kinematic Geometry of Mechanisms, Clarendon Press, Oxford, 1978

[46] Manipulating Industrial Robots-Vocabulary, ISO 8373, 1994

[47] IFToMM Commission A, Terminology for Theory of Machines and Mechanisms, Mechanism and Machine Theory, 26 (1991) 435-539

[48] Karger, A., Classification of Robot-Manipulators with only Singular Configurations, Mechanism and Machine Theory, 30 (1995) 727-736

[49] Karger, A., Classification of Serial Robot-Manipulators with Non-Removable Singularities, Trans. ASME J. Mechanical Design, 118 (1996) 202-208

[50] Karger, A., Singularity Analysis of Serial Robot-Manipulators, Trans. ASME J. Mechanical Design, 118 (1996) 520-525

[51] Kempe, A. B., A Method of Describing Curves of the $n$th Degree by Linkwork, Proc. London Math. Soc., 7 (1876) 213-216

[52] Kieffer, J., Differential Analysis of Bifurcations and Isolated Singularities for Robots and Mechanisms, IEEE Trans. Robotics and Automation, 10 (1994) 1-10 
[53] Kieffer, J. and Lenarčič, J., On the Exploitation of Mechanical Advantage Near Robot Singularities, Proc. 3rd Intl. Workshop on Advances in Robot Kinematics, Ferrara, Italy, (1992) 65-72

[54] Koetsier, T., A Contribution to the History of Kinematics I, Mechanism and Machine Theory, 18 (1983) 37-42

[55] Lazard, D., On the Representation of Rigid-Body Motions and its Application to Generalized Platform Manipulators, Computational Kinematics, J. Angeles et al. (eds), Kluwer Academic, Dordrecht, (1993) 175-181

[56] Lerbet, J. and Hao, K., Kinematics of Mechanisms to the Second Order-Application to the Closed Mechanisms, Acta Applicandae Mathematicae, 59 (1999) 1-19

[57] Litvin, F. L. and Parenti-Castelli, V., Configurations of Robot Manipulators and Their Identification and the Execution of Prescribed Trajectories, Trans. ASME J. Mechanisms, Transmissions and automation in Design, 107 (1985) 170-188

[58] Litvin, F. L., Yi, Z., Parenti-Castelli, V. and Innocenti, C., Singularities, Configurations and Displacement Functions for Manipulators, Int. J. Robotics Research, 5 (1986) 66-74

[59] MacCallion, H. and Pham, D. T., The Analysis of a Six Degree of Freedom Work Station for Mechanized Assembly, Proc. 5th World Congress on the Theory of Machines and Mechanisms, Montreal, (1979) 611-616

[60] McCarthy, J. M., Introduction to Theoretical Kinematics, MIT Press, Cambridge MA, 1990

[61] Merlet, J. P., Singular Configurations of Parallel Manipulators and Grassmann Geometry, Int. J. Robotics Research, 8 (1992) 45-56

[62] Montaldi, J., On Generic Composites of Maps, Bull. London Math. Soc., 23 (1991) $81-85$

[63] Murray, R. M., Li, Z. and Shastry, S. S., A Mathematical Introduction to Robotic Manipulation, CRC Press, Boca Raton, 1994

[64] Nevins, J.L. and Whitney, D. E., Assembly Research, Automation, 16 (1980) 595-613

[65] Pai, D. K. and Leu, M. C., Genericity and Singularities of Robot Manipulators, IEEE Trans. Robotics and Automation, 8 (1992) 545-559 
[66] Panyushev, D. I., Semi-Direct Products of Lie Algebras, Their Invariants and Representations, arXiv:math.AG/0506579

[67] ParalleMIC, Terminology Related to Parallel Mechanisms, http://www.parallemic.org/Terminology/General.html

[68] Pottmann, H. and Ravani, B., Singularities of Motions Constrained by Contacting Surfaces, Mechanism and Machine Theory, 35 (2000) 963-984

[69] Rico Martíez, J. M. and Duffy, J., Orthogonal Spaces and Screw Systems, Mechanism and Machine Theory, 27 (1992) 451-458

[70] Rico Martíez, J. M. and Duffy, J., Classification of Screw Systems I and II, Mechanism and Machine Theory, 27 (1992) 459-490

[71] Roberts, S., On Three-Bar Motion in Plane Space, Proc. London Math. Soc.. 7 (1875) $14-23$

[72] Sagle, A. and Walde, R., Introduction to Lie Groups and Lie Algebras, Academic Press, New York, 1973

[73] Selig, J., Geometrical Methods in Robotics, Springer Verlag, New York, 1996

[74] Simaan, N. and Shoham, M., Singularity Analysis of Composite Serial In-Parallel Robots, IEEE Trans. Robotics and Automation, 17 (2001) 301-311

[75] Stanišić, M. M. and Engelberth, J. W., A Geometric Description of Manipulator Singularities in Terms of Singular Surfaces, Proc. 1st Int. Meeting of Advances in Robot Kinematics, Ljubljana, Slovenia, (1988) 132-141

[76] Stewart, D., A Platform with 6 Degrees of Freedom, Proc. Inst. Mechanical Engineers, London, 180 (1965) 371-386

[77] Sugimoto, K., Duffy, J. and Hunt, K. H., Special Configurations of Spatial Mechanisms and Robot Arms, Mechanism and Machine Theory, 17 (1982) 119-132

[78] Tchoń, K., Singularities of the Euler Wrist, Mechanism and Machine Theory, 35 (2000) 505-515

[79] Tchoń, K. and Muszynski, R., Singularities of Nonredundant Robot Kinematics, Int. J. Robotics Research, 16 (1997) 71-89 
[80] Veldkamp, G. R., Canonical Systems and Instantaneous Invariants in Spatial Kinematics, J. Mechanisms, 2 (1967) 329-388

[81] Wall, C. T. C., Geometric Properties of Differentiable Manifolds, Geometry and Topology, Rio de Janeiro, Lecture Notes in Mathematics 597, Springer, Berlin, (1976) 707-774

[82] Wang, S. L. and Waldron, K. J., A Study of the Singular Configurations of Serial Manipulators, Trans. ASME J. Mechanisms, Transmissions and Automation in Design, 109 (1987) $14-20$

[83] Watson, P. C. A Multidimensional System Analysis of the Assembly Process as Performed by a Manipulator, presented at 1st North American Robot Conf., Chicago, 1976

[84] Wenger, P., Classification of 3R Positioning Manipulators, ASME J. Mechanical Design, 120 (1998) 327-332

[85] Wenger, P. and El Omri, J., Changing Posture for Cuspidal Robot Manipulators, IEEE Int. Conf. on Robotics and Automation, Minneapolis, (1996) 3173-3178

[86] Whitney, D.E. and Nevins, J.L., What is the RCC and what can it do?, Robot Sensors, Tactile and Non-Vision, ed. A.Pugh, IFS Publications, (1986) 3-15

[87] Zlatanov, D., Bonev, I. A. and Gosselin, C. M., Constraint Singularities of Parallel Mechanisms, Proc. IEEE Int. Conf. on Robotics and Automation, Washington, DC, (2002) 496-502

[88] Zlatanov, D., Fenton, R. G. and Benhabib, B., Singularity Analysis of Mechanisms and Robots via a Motion-Space Model of the Instantaneous Kinematics, Proc. IEEE Int. Conf. on Robotics and Automation, San Diego, CA, (1994) 980-985

[89] Zlatanov, D., Fenton, R. G. and Benhabib, B., Singularity Analysis of Mechanisms and Robots via a Velocity-Equation Model of the Instantaneous Kinematics, Proc. IEEE Int. Conf. on Robotics and Automation, San Diego, CA, (1994) 986-991 